\title{
versants
}

\section{Il languorino}

Giorgio FALCo \& Sabrina RagucCI

Milano

Abstract: Una digressione sul rapporto tra testo e immagine: dal segno al linguaggio, l'influenza di Atget su Evans, di Evans su Frank, dal modernismo anti-narrativo fino al manifestarsi della scrittura nell'immagine. Gli autori ripercorrono alcuni loro lavori fototestuali.

Keywords: Eugène Atget, Walker Evans, Berenice Abbott, modernismo, Robert Frank, Jean-Luc Godard, Jim Dine, testo-immagine.

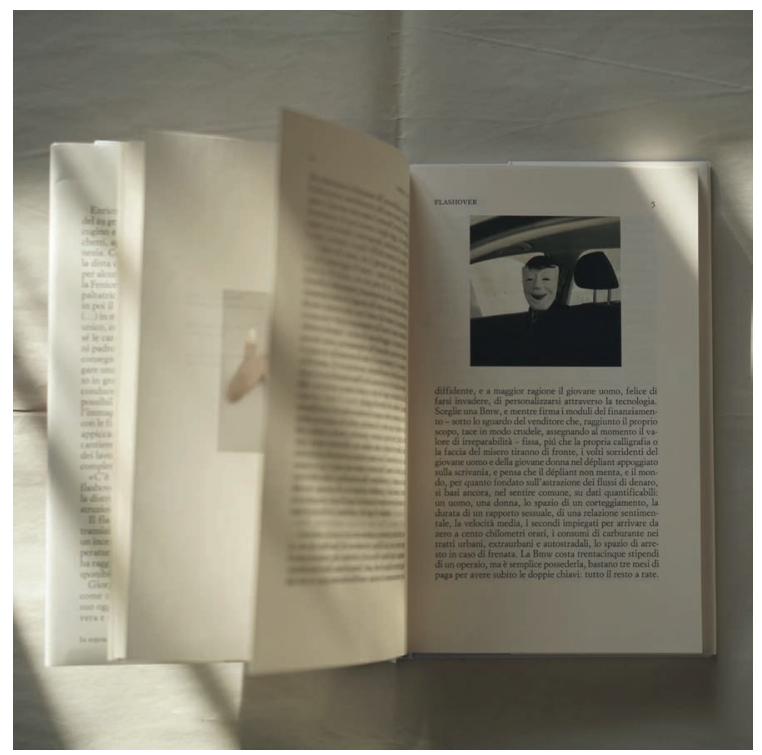

I

Il silenzio si relaziona alla parola e, tra una parola e l'altra, la scrittura respira; la fotografia si relaziona al silenzio, è muta e allontana in modo irreversibile la realtà, tramutandola in traccia; ma l'immagine indica il diritto d'esistenza dell'altro e così la fotografia si apre al dialogo, sebbene abiti ormai una terra sovraccarica di lettere dell'alfabeto; la relazione tra scrittura e fotografia non dovrebbe generare una favola, forse un contrappunto.

I Le immagini del saggio sono tratte dal romanzo Flashover (Falco-Ragucci 200o).

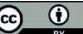


Il contrappunto, dice Godard, è la disciplina della sovrapposizione delle linee melodiche. Le linee melodiche non hanno bisogno di essere identiche. Estranee l'una all'altra, non ostacolano la composizione, ma si tengono insieme: solo nell'armonia gli accordi producono melodie (Le Livre d'image, 20I8).
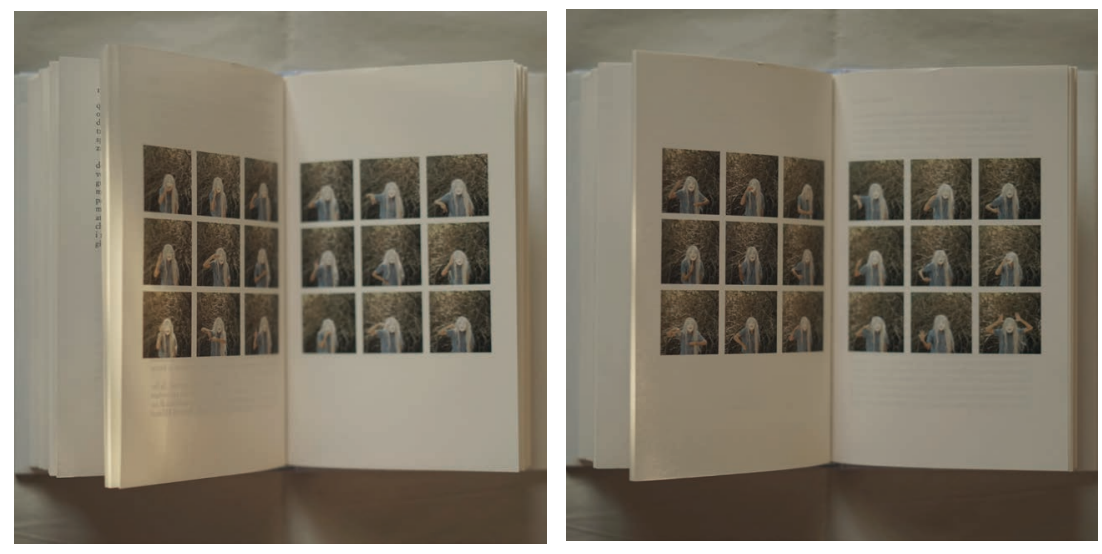

\section{2}

Gli uomini prima di Lascaux come parlavano e di conseguenza cosa pensavano (Kallir 1994)'? Sappiamo poco del significato reale delle immagini ritrovate fuori e dentro le caverne: le immagini per noi non sono più magia, comunicazione tra uomo e spiriti che dominano la natura. Del resto, le immagini non hanno mai voluto parlarci con chiarezza; certo, possono indurre al sentimento, dalle caverne alle metropoli ne intuiamo la potenza, ci inducono a comprare abiti e automobili, eppure ne rimuoviamo ogni aspetto estraneo, le traduciamo, quasi sempre parliamo sopra le immagini: l'arte contemporanea parla a pochi, la pubblicità parla a tutti, ma poco importa. Anche la scrittura (la scrittura-segno in sé) è stata considerata un'arte e, in effetti, le lettere dell'alfabeto per alcuni non sono altro che «immagini decadute» (Kallir 1994: 37). Può capitare allora, di svegliarsi una mattina e con spirito infantile chiedersi: dove è sprofondata la lingua preistorica comune all'intero genere umano? Vive da qualche parte nell'inconscio collettivo? Da qualche parte ha germinato - nelle lingue successive - un'unità psicogenetica tra le genti? Questo è quanto suggerisce il caso dell'ufficiale di origine austriaca, Alfred Kallir, inglese di nazionalità, decifratore e abi-

2 È nata prima la parola o la rappresentazione? Pare che questo sia un aspetto controverso, la risposta dipende in parte da quale sia la testimonianza visiva del pensiero ritenuto effettivamente il primo stadio della scrittura. 
le agente del controspionaggio militare britannico, che durante la Seconda Guerra Mondiale incominciò a interessarsi alle lettere dell'alfabeto, analizzando l'effetto straordinario che la lettera V, usata da Winston Churchill, aveva sugli inglesi, come simbolo di vittoria.

August Sander amava sottolineare, durante le conferenze (Sander 193I), quanto la fotografia fosse universale, l'equivalente moderno di una lingua visiva originaria anteriore alla scrittura, fatta di segni e dal carattere immediato: per questo motivo August Sander si riferiva spesso ai geroglifici egizi. Il riferimento ai geroglifici è stato un tema ricorrente in tutta la letteratura fotografica del XIX secolo, tanto quanto l'evoluzione della specie umana correlata all'evoluzione del linguaggio. E tuttavia, l'uomo non ha solo parlato o guardato, deve avere anche ascoltato, come probabilmente fa ogni specie, flora o fauna, che desideri sopravvivere per un periodo di tempo molto lungo. Forse il segreto che presuppone la necessità di una qualsiasi lingua potrebbe anche essere il semplice desiderio di essere ascoltati. Questa è la prima e immediata relazione tra due linguaggi: la scrittura che parla e guarda, la fotografia che ascolta e vede ciò che è invisibile all'uomo. La fotografia che usa il teleobiettivo per accentuare la verità, e la interroga sconfessandola.

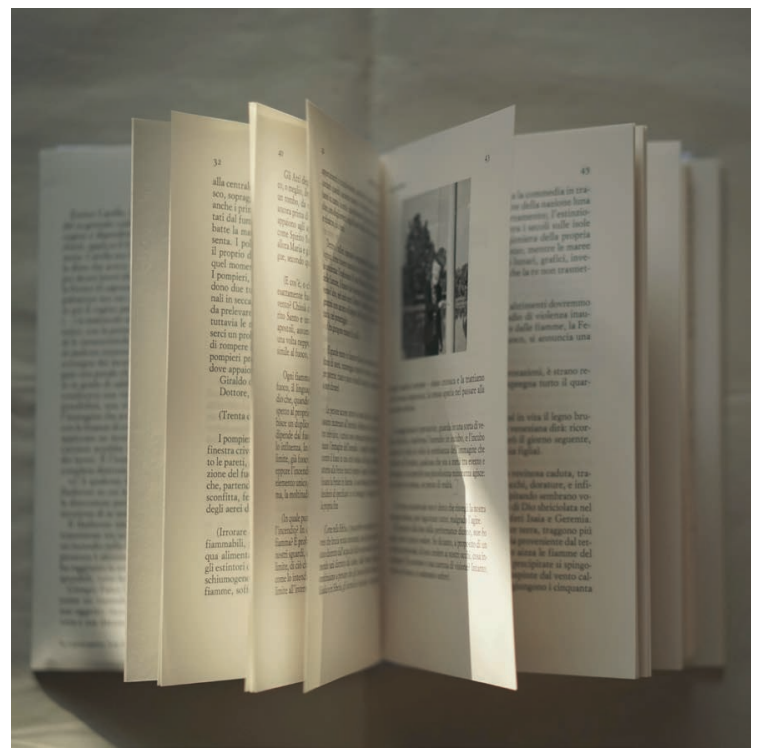

Il bambino nei giorni di apprendimento della lingua scritta vede la parola albero, e osserva la rappresentazione di un albero disegnato, così pensa all'albero dietro casa, e finalmente la rappresentazione dell'albero e della 
lettera $\mathrm{A}$, ora sono associate all'albero dietro casa. L'albero della realtà è detto due volte, trasformato in immagine e in scrittura, nell'abbecedario venduto - per quattro soldi - da Pinocchio; l'albero dell'abbecedario è simile nel disegno e diverso nella scrittura all'albero dietro casa, tuttavia il bambino può affidarsi all'esperienza e al linguaggio della madre per sapere cosa si intende con la parola albero. E allora, si chiederà il bambino, qual è l'immagine per il significato della parola potere? Secondo Socrate non vi sarebbero né parole né immagini, se queste sapessero riprodurre le cose così fedelmente da raddoppiarle (Platone, Cratilo).

Un'attenzione reverenziale alle parole - pari solo alla sfiducia nei confronti di ciò che le parole erano diventate - la si è trovata nella fotografia modernista. Nel 1933 Brassaï pubblicava, su Le Minotaure, le immagini dei graffiti sui muri di Parigi, una serie iniziata nel I929. Si trattava di segni concisi che raffiguravano le origini della scrittura. Il mondo borghese si esprimeva tramite la lingua della pubblicità riecheggiante nelle strade, ridondante nelle parole dei politici, nella poesia ironica fatta di ritagli di giornali, di collage che tentavano di stravolgere la serietà ingannevole della promessa commerciale. La fotografia in bianco e nero, fino alla generazione di Walker Evans, era allo stesso tempo frivola e cabalistica e aveva in James Joyce il nume tutelare di questa nuova religione dell'arte (Evans I998: I). Il giovane Walker Evans era cresciuto con la venerazione per alcuni Alti Modernisti, devoto alla letteratura, tanto quanto in seguito l'uomo si era votato alla forza ostinata dell'estetica moderna, presente in tutte le sue opere. Per la maggior parte, i fotografi, gli artisti, i critici e i curatori vissuti intorno a Evans hanno difeso a lungo la volontà di una posizione dell'arte anti-narrativa, contraria a una traduzione letterale e letteraria, in antitesi sia alla fotografia giornalistica, sia a quella specificatamente concettuale, non sempre adatta a una grammatica e sintassi visiva, per dirla con Berenice Abbott (I94I: 93). Tuttavia, proprio Berenice Abbott e la critica d'arte Elizabeth McCausland - nel secondo tempo della loro vita, quello concesso agli artisti per andare altrove dopo il consolidamento - si sono sempre più interessate all'integrazione tra testo e immagine. Segni lessicali, graffiti e manifesti inseriti nella composizione rivelavano il desiderio collettivo condiviso di ricreare una lingua a parte, una sorta di lingua-rebus che richiedesse una nuova leggibilità; questo in particolare si augurava Elizabeth McCausland: un rapporto paritetico di relazione per il futuro dell'immagine e della scrittura, «leggere fotografie e parole insieme» (McCausland 1942: 2785). Nel 1973, Walker Evans aveva iniziato a fotografare - a colori, non in medio o grande formato, come era solito fare, ma con una macchina che presumibilmente considerava quasi un giocattolo, la Polaroid SX 70 - sillabe selezionate e ritagliate da cartelli diversi. Non mancavano, nel suo ricorrente alfabeto, W e E, iniziali del nome e del cognome, alla maniera di un pittore dalla firma riconoscibile. Le scritte 
avevano una vita propria nelle immagini di Walker Evans già dagli anni Trenta, la scrittura trasformava il soggetto in una sceneggiatura e, come sempre nel caso di Evans, in riflessioni sull'arte. Raccoglieva cartelli, segni, tracce, oggetti agli angoli delle strade o li acquistava in negozi di antiquariato, e qualche volta esponeva le sue reliquie vicino alle rappresentazioni fotografiche che ne aveva tratto. Evans è morto nel 1975, prima che il suo dizionario potesse essere completato. Lee Friedlander, in Letter from the People, ha continuato ciò che il suo amico e mentore aveva cominciato, e qui - come succede quando un artista prende il testimone dal suo predecessore - si è aggiunto un altro mondo, sembrava che la lingua si moltiplicasse scrivendosi da sola. La sequenza delle immagini partiva dalla lettera A. Sfogliando le pagine - messe in sequenza da Friedlander - le voci hanno cominciato a discorrere, e la parola arte non è rimasta a lungo senza risposta, in una diceva chiaramente: «fa male», e ovviamente un cartello avvertiva: «Attenzione. Suonare Ioo volte se necessario» (là dove non c'era nessun campanello), fino alla rivelazione: «tutti i giorni le telefono - tutte le notti me la sogno». L'enigma irrisolvibile è sempre stato lo stesso e irriducibile: di cosa sta parlando la fotografia di Lee Friedlander? Forse qui potremmo aggiungere un bel decidi $t u$. Friedlander ha fotografato singole lettere e parole che appaiono su edifici e insegne; il suo interesse era rivolto alla condizione di reperto ritrovato, come un archeologo della cultura popolare, un catalogatore di lettere, un collezionista di segni, un salvatore dell'esausto, un creatore di un nuovo ordine. Le immagini non volevano essere semplici riflessi delle emozioni dell'autore, se non - al limite - come effetto secondario.

Anche molte fotografie di Aaron Siskind mostrano tracce calligrafiche dell'attività umana e alcune raffigurano graffiti e slogan politici che sono stati dipinti o cancellati. Queste immagini suggeriscono i blocchi e le complessità della comunicazione umana, sebbene nel caso di Siskind le implicazioni siano sociali e politiche. Parole scritte a mano o graffiate direttamente sul negativo si trovano nelle fotografie di Robert Frank degli ultimi quarant'anni della sua vita. In opere come Sick of Goodby's (1978) e Moving Out (I984), Frank sovrapponeva informazioni pittoriche con frasi e espressioni proprie. Il fotografo del leggendario libro The Americans e il regista di film fuori dal circuito, riuniti in un'unica identità morale: il solo obiettivo di una macchina fotografica non poteva ormai esprimere - senza l'incursione della parola nell'immagine - il dolore dell'uomo nel mondo; così Frank ha inserito prove dei suoi sentimenti dipingendo parole sugli specchi o intorno alle fotografie, scritture frammentate, apparentemente spontanee, autobiografiche e confessionali. Questo è anche il caso dell'artista e fotografo ucraino Boris Mikhailov, nel suo Unfinished Dissertation. In un solo mese, durante l'inverno del i984, accumulando una palese critica all'Unione Sovietica e uno sguardo che usciva dai confini di amici e famigliari, ha scattato diverse 
centinaia di istantanee e solo dopo avere finito di raccogliere le immagini le ha incollate su fogli economici e ha iniziato a scriverci sopra; il più delle volte, riempiva i margini attorno ai quattro lati delle fotografie, un insieme di note e commenti autobiografici sul suo lavoro, inserendo talvolta testi interpretativi ispirati alla filosofia, alla teoria dell'arte e della scienza. Sul retro dei fogli si poteva leggere una sorta di titolo: «Manoscritto di Mikhailov (1998)».

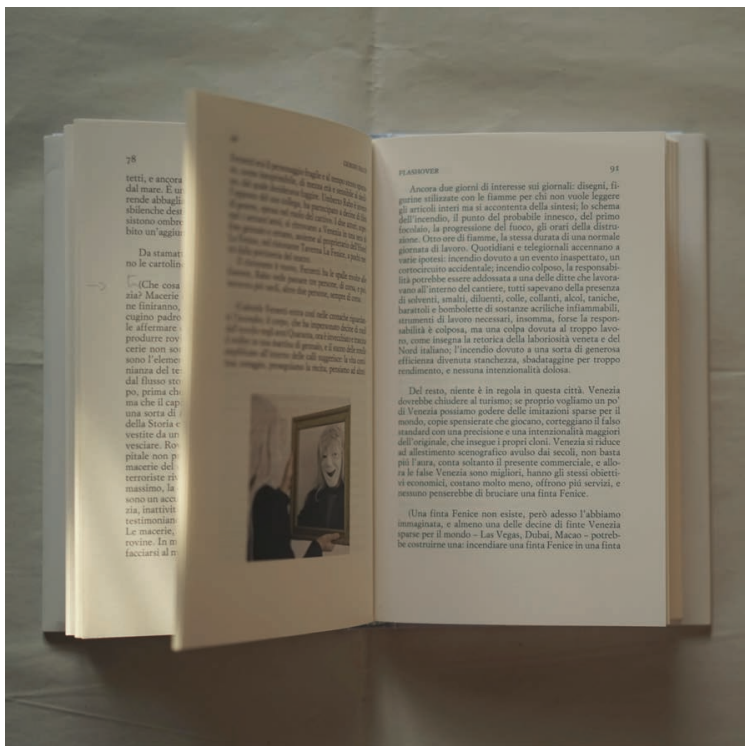

A differenza della pittura, la fotografia rimane un frammento che non può essere compreso e visto da solo, anche se in realtà, diceva Brecht, solo il frammento porta il marchio dell'autenticità perché è il più vicino alla funzione più interna dell'atto di produzione, una produzione che corrisponde alla respirazione, al semplice fatto di esistere vissuto solo dalla coscienza ( $L e$ Livre d'image, 2018).

4

Le scritte sui muri riportano in superficie fasi passate della realtà, comunicano da una dimensione parallela, il passato che travalica nel presente e, qualche volta, parla proprio a noi. Anche questo è linguaggio fotografico. «Le scritte sui muri sono così: crude e dirette. Avremmo potuto guardare la televisione o ascoltare il videofono [...] - per sempre, forse - senza scoprirlo. Senza che ci venisse detto così a brutto muso: Io sono vivo, voi siete morti» (Dick 2008: 136). 
Quando ancora pensava di diventare uno scrittore, Walker Evans era stato attratto dalla cultura francese. Alla fine della carriera diceva di se stesso: da giovane sono stato un anti-americano. Partito per la Francia nel 1926, vi era rimasto un anno, e lì, grazie a Berenice Abbott, aveva scoperto Eugène Atget e quello che, in seguito, avrebbe definito lo stile documentario lirico. Ma era necessario occuparsi dell'America, come era accaduto a Edward Hopper: diventare un autentico artista americano. Il concetto stesso era nell'aria. Lincoln Kirstein aveva guidato Evans da Baudelaire a Whitman, uno stile trasparente, senza arte, senza alcun ornamento; da Eugène Atget a Matthew Brady, dal romanticismo europeo al puritanesimo americano, e fino all'architettura, alle fotografie delle case vittoriane realizzate nei dintorni di Boston, al fondamentale American Photographs. Tanto che già nel I93I Evans scriveva che l'America è il focolare naturale della fotografia e anche il suo lavoro alla Farm Security Administration, campagna di ricerca e archiviazione sovvenzionata dal governo negli anni del New Deal, voleva essere esclusivamente arte, disinterested. Tuttavia, Evans si riferiva allo stile documentario per intendere una sorta di oggettività attiva, che non pretendesse di restituire le cose in quanto tali: non solo presenza, ma lingua della realtà, per dirla con James Agee (Agee-Evans 2002: 236). Così Evans sarebbe stato uno dei primi fotografi della sua epoca a impegnarsi in un lavoro meta-fotografico, diventando punto di riferimento per gli artisti della Pop Art, dell'arte concettuale e anche della Picture Generation, nonché uno degli artisti più citati del secolo scorso: da Andy Warhol a Dan Graham, da Sherrie Levine a Jeff Wall.

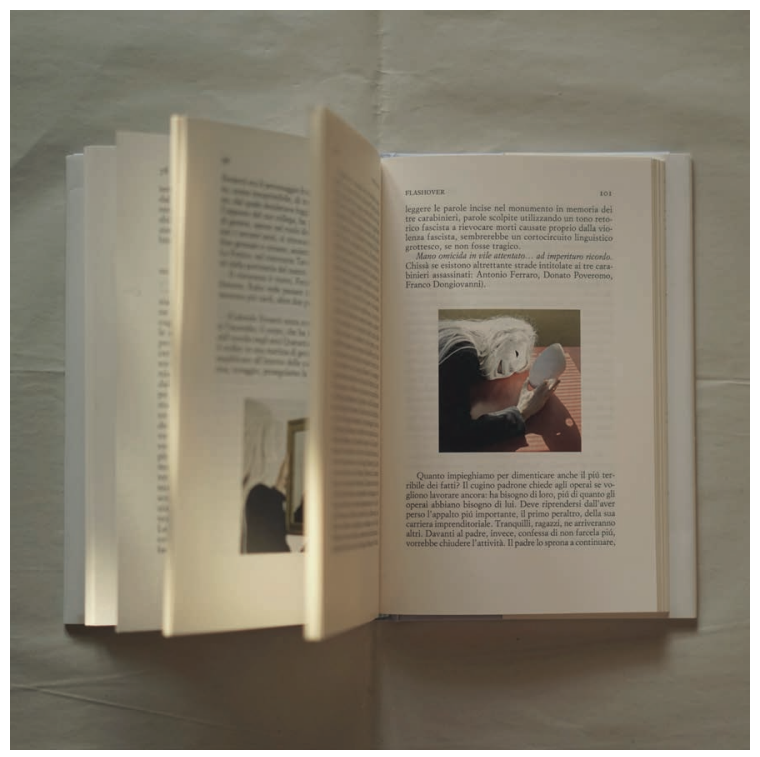


Ferdinand, "Pierrot le fou" di Godard, sigaretta in bocca, adagiato nella vasca da bagno, legge dalla Storia dell'arte di Elie Faure un brano su Velázquez, che alla fine della sua vita non dipingeva più le cose definite ma quello che c'è tra le cose. La frase su Velázquez in apertura è anche il progetto estetico di Pierrot-Ferdinand-Godard, ribadito in una battuta del film: «Ho avuto un'idea per un romanzo. Non descrivere più la vita della gente, ma soltanto la vita, la vita da sola. Quello che c'è fra la gente, lo spazio, il suono, i colori. Bisognerebbe giungere a questo». Joyce ha tentato, dice il personaggio, ma si dovrebbe fare meglio. Joyce, già nume tutelare di Evans e in seguito di Godard. James Joyce e Henry James spesso definiti da Walker Evans inconsciamente fotografi, quasi parenti di Walt Whitman. In Foglie d'erba, sosteneva Evans, ogni cosa è letteralmente fotografata, niente poetizzato. Desideroso di emulare il sacerdote del sole, Whitman aveva composto un poema, che a tratti sembrava una lunga didascalia per un catalogo di fotografie in costante evoluzione. «Guardai i bei raggi di luce partirsi centrifughi dall'ombra della mia testa sull'acqua, guardai le colline velate di vapori a sud-ovest, guardai il vapore disperdersi in bioccoli tinti di viola. Guardai verso la baia inferiore, per osservare i vascelli in arrivo [...]» (Whitman 1977: I07).

Alla fine, Godard, il suo romanzo, lo ha realizzato nel 20I8, un romanzo tutto composto di parole e immagini, prelevate da scrittori, poeti, filosofi, registi (non manca una certa influenza di Robert Frank, di Nicholas Ray, di Anne-Marie Miéville, sua compagna): Le Livre d’image. «Non c'è niente di più comodo di un testo, e non c'è niente di più comodo di una parola in un testo. Avevamo solo un libro da mettere in un libro, come faremo quando nel libro, in un libro, dovremo mettere la realtà e al secondo grado: quando bisognerà mettere realtà nella realtà?» (Le Livre d'image, 20I8). L'inconscio è davvero più accessibile tramite le immagini? Il potenziale narrativo, che è implicito nella natura dell'immaginario, non si evolve mai realmente, ma dice di per sé. Oppure è un continuo suggerire come nella difficile relazione tra fotografia e poesia, ut pictura poësis, laddove immagini e poesia dovrebbero svolgere funzioni simili nel mondo, tutta immaginazione e metafora, tanto da avere convinto Jim Dine - dopo essere entrato in contatto con Letter from the people di Lee Friedlander ${ }^{3}$ - a combinarle nel suo lavoro. La poesia riflette il funzionamento dell'occhio interno dell'artista. La fotografia è legata al mondo visibile e alla verosimiglianza, e quindi raggiunge la condizione di metafora solo attraverso i poteri associativi del geroglifico stesso.

3 «Lee Friedlander came - they wanted him to look at the camera - and he was very mean to me, dismissive. The implication was, "You're not a photographer. What right do you have to do this?"» (Dine 2003: 37). 
Tuttavia, come tutti i linguaggi simbolici, poesia e fotografia dipendono entrambe dal sottrarre racconto e dall'aggiungere intensità, dalla possibilità di lasciare intravedere la verità oltre la superficie del mondo. Sembrerebbe che, per Dine, la fotografia fornisca un inventario impersonale e quasi automatico di oggetti, quindi il suo fascino sarebbe limitato. Almeno, così è stato per lui, fino a quando ha scoperto che poteva letteralmente inserire la scrittura nella fotografia, come forma di stenografia per sogni, pensieri di veglia, sentimenti primordiali: frammenti di poesie in un'eco surrealista. Le sue parole, fissate alla stregua di una traccia su un positivo Polaroid 20x25, hanno acquisito lo stato di fatto del documento, segno indicativo, insomma, la cosa in sé dei fotografi; ma qui, appunto, incluso nello statuto di documento, si aggiungeva la registrazione stessa dell'atto dello scrivere. Sebbene l'otturatore possa aprirsi solo per una frazione di secondo per registrare un'immagine, l'immagine conteneva una più ampia distesa di tempo: rappresentava l'atto della mano dell'artista mentre scrive, cancella e riformula le parole che noi vediamo (Dine 2003: 24).

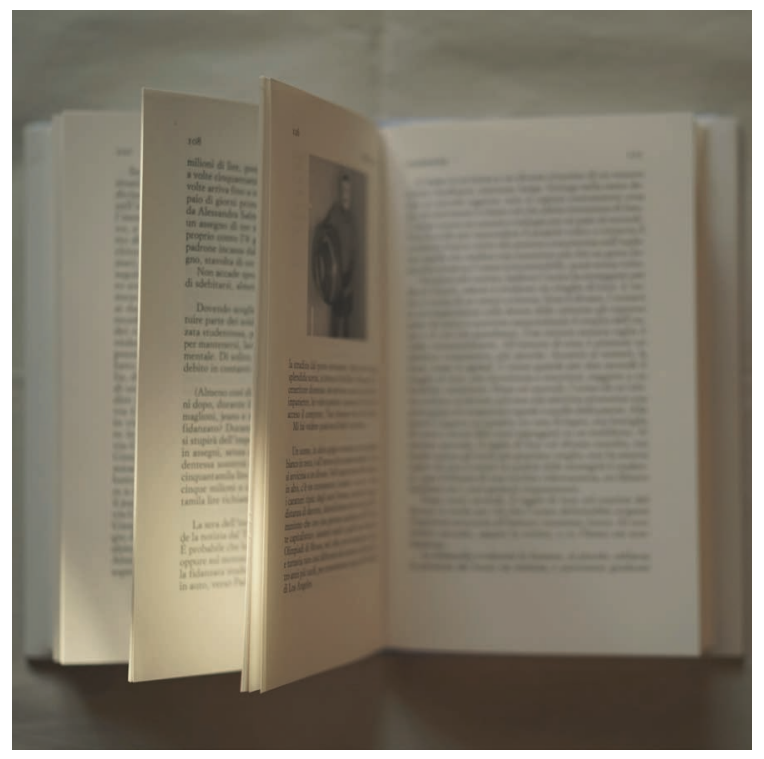

Qualcuno ha detto che nelle arti non c'è progresso ma proseguimento di una linea, che va da una generazione all'altra per serbare il ricordo di una certa esperienza. Un piccolo languore è la condizione che, per noi, giustifica l'esistenza di un fototesto. Ma non consideriamo questa condizione come il manifestarsi o l'aggravarsi di una sensazione di vuoto, insomma, il languore 
come stato di avvilimento che giunge fino alla prostrazione romantica, o peggio, allo struggimento di un certo sentimentalismo contemporaneo. Il nostro piccolo languore è qualcosa di gioioso, la sensazione di leggera euforia che si provava, da bambini, poco prima del pranzo: il languorino. A noi, bambini degli anni Settanta, ricorda il sottofondo di suoni, il concerto di piatti e bicchieri e posate e pentole, di acqua calda rovesciata nello scolapasta, di luce che entra dalla finestra e illumina e seziona l'ultimo vapore, poco prima che dallo scolapasta il vapore si disperda in cucina. Il languorino è qualcosa di gioioso e ludico e traumatico: è l'attesa, l'avvento. Quando lavoriamo a un fototesto, cerchiamo di creare e mantenere in vita quel languorino di fine Novecento. Quindi, per quanto ci riguarda, l'unione tra fotografia e letteratura inizia dalla consapevolezza della presenza e dell'assenza.

Ogni volta che ci poniamo davanti a un'immagine, ci chiediamo: sentiamo davvero, a questo punto, il languorino? Oppure inserire un testo, inserire un'immagine, risponde a un'esigenza di accumulo bulimico, spesso troppo lontana dalla sacra dimensione di «il Dio è sempre geometra»? o - se si preferisce - del «qui appare la forma?»4. E naturalmente per forma, intendiamo la perfetta necessità dei due linguaggi.

Inserire fotografie in un romanzo o in un testo letterario, non significa automaticamente fare di quell'opera un fototesto. Molto spesso, scrittori, poeti e fotografi che si cimentano nel fototesto ricordano disorientati atleti di biathlon. Come è noto, il biathlon è lo sport costituito dall'abbinamento di due discipline: lo sci di fondo e il tiro con la carabina. Se, durante la gara, ti fermi in mezzo al bosco e cominci a sparare a casaccio, al posto di aspettare l'arrivo al poligono, spari inutilmente. Ovviamente il biatlhon è più semplice da leggere rispetto a un fototesto. Infatti, con il fototesto puoi anche sparare nel punto esatto, ovvero al poligono, e non ottenere nulla: puoi essere il miglior fondista del mondo, ma se sei scarso con la carabina, il risultato sarà mediocre; puoi essere il miglior tiratore del mondo, ma se sei scarso con gli sci di fondo, il risultato sarà mediocre. Ciò che conta è che la qualità di scrittura sia in dialogo con la qualità del linguaggio visivo e viceversa. Ciò che conta è rispondere con assoluta onestà a una semplice domanda. Senti il languorino oppure puoi evitare di inserire le fotografie?

\footnotetext{
4 "'Aèi ho theòs geōmetreî". Questa iscrizione greca letta sul frontone di una casa antica, mi torna sempre in mente, perciò la traduco anche per me: "Il Dio è sempre geometra" (= misura la terra). Oppure per chi voglia eliminare dal gioco il "Dio", o la parola straniera, e perfino il "sempre", può bastare anche solo: qui appare la forma» (Handke 20I4: 80) (parlando di toilette, à vrai dire). Aèi ho theòs geōmetrê̂, "Dio geometrizza sempre" - Platone. Plutarco elaborò que-

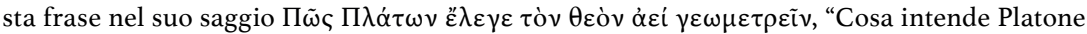
quando dice che il dio applica geometrizza ogni cosa?". Sulla base della suddetta frase di Pla-

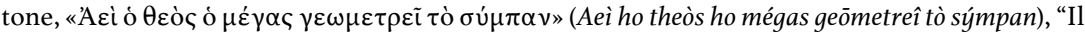
grande Dio geometrizza sempre tutto".
} 
La maggior parte dei migliori romanzi di quest'ultimo secolo non ha avuto alcun bisogno di immagini e alcuni sono opere ineguagliabili.

Riprendiamo il concetto di trascendente più volte evocato da Walker Evans. Nell'introduzione a Il libro rosso di Jung (2012: 82) si può trovare un passaggio molto adatto al tipo di relazione che si innesca in un lavoro di accostamento di parole e immagini: si tratta di due strade legate da un rapporto di compensazione che ne fa l'una il principio regolatore dell'altra. In tal modo entrambe concorrono ad attivare la funzione trascendente che si esprime dall'unificazione dei contenuti consci e inconsci.

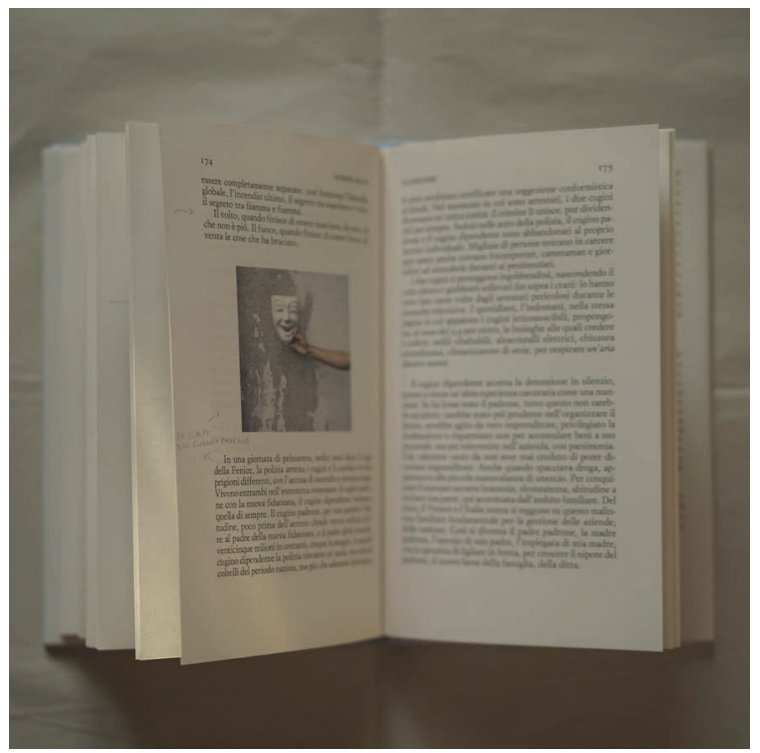

In ambito artistico la scrittura abbinata all'immagine è stata ampiamente indagata, qualche volta risolta togliendo dall'esito proprio l'immagine e lasciando solo il testo. Anche noi, nei romanzi, spesso abbiamo tolto l'immagine fotografica ${ }^{5}$.

La combinazione - nei prolifici anni Sessanta - aveva moltissime espressioni, persino tramite la poesia visiva o la poesia concreta o la pittura che cancellava le parole (dopo una dittatura, cosa si poteva fare?). All'epoca il

5 Nel primo romanzo, Il medesimo mondo, di Sabrina Ragucci (2020) l'unica fotografia presente è quella della copertina. Anche L'ubicazione del bene, di Giorgio Falco (2009), è un libro attraversato da riferimenti fotografici, ma il libro è stato impaginato senza alcuna necessità di inserire le immagini, nonostante Falco-Ragucci abbiano attraversato ed edificato la «Cortesforza» di Falco che appare nella serie fotografica The Collared Dove Sound (2004-2012) molti anni prima della pubblicazione del libro per Einaudi. 
poeta prelevava l'estetica, l'artista la ridondanza della metrica. Di recente, si è consolidata la definizione fototesto - o iconotesto - nonostante la supremazia della parola sull'immagine, quest'ultima spesso considerata riempitivo, corredo. E allora, ben venga il fototesto, certo; ma la letteratura viene davvero prima della fotografia? In Italia tale ipotetica supremazia deriva dalla percezione dei lettori, i quali hanno spesso un'abitudine al romanzesco, sia esso fatto di parole o di immagini.

La conseguenza dell'inevitabile confusione è l'equivalenza di libri considerati fototesti soltanto perché all'interno compaiono immagini o parole gettate nella mischia, allestite con una grafica ammiccante. Beh, così come non serve un pennello grande per fare un grande pennello, allo stesso modo non bastano le fotografie in un libro per trasformare l'opera in un fototesto, e nemmeno una lista di parole in un libro fotografico può trasformare una sequenza di fotografie in iconotesto (meglio Le Livre d'image, allora!).

Se la poesia ha bisogno di una forma, anche la fotografia, nell'arte, funziona allo stesso modo. Se - come sostiene Mark Strand - tutta la poesia è formale ed esiste entro certi limiti, limiti che sono ereditati dalla tradizione oppure limiti che la lingua stessa impone, allora questi limiti esistono a loro volta all'interno della concezione che il singolo poeta ha di cosa sia o non sia una poesia (Strand 2019). Questo è esattamente ciò che accade con il linguaggio di un artista che non è mai svincolato da una tradizione. Del resto, forma è una parola poderosa, dice ancora Strand, proprio per questa ragione: struttura ed essenza paiono unificarsi, tanto quanto la disposizione delle parole o delle immagini e il loro nuovo significato.

E ora perdonerete se parliamo un po' di noi attraverso il fototesto Condominio Oltremare ${ }^{6}$ e con un accenno all'ultima opera, Flashover, pubblicata nel settembre 2020, per Einaudi, nella collana "Frontiere".

Condominio Oltremare è un libro che si può leggere su più livelli. È un'opera che parte dalla sedimentazione di sopralluoghi invernali cominciati nel 1993, quando entrambi eravamo ancora studenti d'arte, e combinavamo immagini e prose tutte, per fortuna, sistematicamente distrutte, prima di iniziare il vero Condominio. L'effettiva durata del lavoro visivo, pubblicato nel 20I4, è databile infatti dal 2006 al 20I4, un processo di lavorazione che è stato anche una lunga verifica. A partire dalla semplice annotazione che le amate casette piccolo borghesi disegnate ossessivamente come fossero il nostro corpo; i luoghi abitati e guardati da questa prospettiva (strutture circondate dall'acqua) conservavano sia il desiderio incosciente di essere cullati in un mare tiepido che una relazione plastica tra figura e sfondo, tra uomo e habitat, tra uomini e luoghi abitabili anche se, di fatto, deserti. In questo

6 Cfr. Falco-Ragucci 20I4; esposto nel 20II alla Biennale di Venezia, con il titolo The Italian East Co(a)st. 
specifico caso di collaborazione e dialogo, è nato prima il lavoro visivo. Durante gli otto anni, per una sorta di naturale predisposizione alla Land Art insita nella fotografia, sono stati riempiti non solo rulli I20 Kodak Portra pellicola a colori, I6o asa, ma anche taccuini di appunti. Abbiamo cercato in modo funzionale, solo apparentemente distaccato, di riportare all'interno della frantumazione alcuni elementi decorativo-architettonici; lo abbiamo fatto costruendo entità visive anti-narrative, ma unificanti (ovvero, tramite la serie): quello che è sembrato un continuo ritorno nello spazio ha attraversato l'intrattabile realtà nel tempo ed è proprio in questo ambito che ci si è ritrovati ad affrontare, tra immagini e parole, un ritorno desiderato ma impossibile.

Giorgio Falco nomina la realtà e la trasforma; la fotografia, per paradosso, può, grazie alla presenza della letteratura, disinteressarsi della realtà e, per dirla con Giuseppe Verdi, inventare il vero. La fotografia ribadisce: questo luogo non è come lo vedete, nasce dalla differenza di due linguaggi, fotografia e scrittura, entrambi capaci di mentire, due linguaggi che si affrontano e si rafforzano, due bugie che sommandosi diminuiscono e diventano una verità. Del resto, laddove c'è un atto creativo esiste anche una fase di selezione. Quasi tutta la parte visiva di Condominio Oltremare riguarda gli anni Settanta. Questa scelta implica che gli imprinting visivi dell'infanzia degli autori, condensati nel protagonista, siano coerenti con l'impostazione e l'omaggio modernista e minimalista da Dan Graham a Lewis Baltz, anche se la distanza dai padri si esplicita proprio in quello sguardo sbagliato sul futuro, in quelle case, definite fredde dai sociologi, abitate in media pochi giorni all'anno, proprio tra casa e casa dove alberga l'aria salmastra che corrode, e si palesa l'evidenza dell'assenza, della morte differita.

Quando Andrea Cortellessa ha proposto di fare un libro per la collana "fuoriformato", abbiamo pensato che fosse arrivato il momento di pubblicare quel lavoro in forma di romanzo. Il testo è stato scritto in poche settimane grazie alla sedimentazione precedente: scritture reciproche, discussioni a lato delle immagini.

Condominio Oltremare è fin dalle origini - origini che comprendono un lungo cammino a piedi - un fototesto, ed è un fototesto con un incipit multiplo. Il primo incipit è un documento vero, l'appello del sindaco di Cervia, datato I873, affinché i turisti passino le vacanze proprio a Cervia. Il secondo incipit è visivo; all'appello del sindaco segue una sequenza di immagini raffiguranti la pineta di Cervia; sono immagini degli anni Zero del xxi secolo ma senza alcuna traccia contemporanea; le immagini, se fossero in bianco e nero, potrebbero essere l'estratto di un Ottocento farlocco. Dopo questa sequenza c'è un altro lungo incipit, scritto con uno stile impersonale, da voce documentaria, brano in cui si fa la storia di quei luoghi, dall'antichità al petrolchimico, dagli uomini delle berline nere al turismo, dal mare alle vil- 
lette edificate da Michele Sindona e da Mario Gardini, zio di Raul. Terminata questa parte e dopo una specifica sequenza visiva, inizia il libro vero e proprio, ovvero il diario di un quarantacinquenne milanese che, alla morte dei genitori, si trasferisce in un appartamento all'ottavo e ultimo piano di un palazzo edificato sulla spiaggia, a pochi metri dal mare: il Condominio Oltremare, appunto.

In realtà, un Condominio Oltremare, ai Lidi Ferraresi, in quel punto, non esiste, ma proprio per questo motivo diventa tanto più rappresentativo.

L'io narrante è l'unico essere vivente del palazzo: vi arriva in un lunedì di gennaio, poco prima del tramonto. Manca in quel luogo da ventisette anni. Stringerà tra le mani oggetti che pensava di aver dimenticato: le ultime scarpe da minorenne, il termometro che dopo ventisette anni segna ancora i gradi dell'ultima febbre da adolescente. Le immagini si alternano continuamente al testo, non spiegano nulla, ma contribuiscono a un effetto straniante di realtà. Le fotografie ritraggono sempre gli esterni: finestre, porte, angoli di case costruite negli anni Sessanta e Settanta; e così, quando il protagonista scende dall'ottavo piano e cammina in quei luoghi deserti, spiato dalle case, incontra le fotografie, incontra le architetture dell'epoca di Sindona e solo quelle, perché il resto è stato omesso - in quanto osceno - tagliato fuori dal quadrato, dalla geometria dell'autrice che conosce quei luoghi dalla sua infanzia. Sono le case che il protagonista vedeva quando era bambino e ragazzo, fino ai diciotto anni. Il protagonista è quindi prigioniero del libro.

Lui stesso è una sorta di Virgilio che conduce il lettore nella landa desolata dei Lidi Ferraresi in inverno; sempre ritratto in lontananza o di spalle è un personaggio anonimo, qualsiasi, che a piedi attraversa lo spazio, il luogo dell'incontro, dello scontro, del trauma. La figura del protagonista rappresenta solo idealmente un misto di entrambi gli autori, qualcosa che ci riguarda (non c'è mai autobiografia diretta nel Condominio). Il protagonista è un Virgilio alla ricerca di una necropoli etrusca che non si trova, la reale necropoli di Spina. La necropoli non si trova perché entrambi i protagonisti, letteratura e fotografia, la abitano. Vivono in una vera necropoli fin dall'infanzia, sono già lì. Le fotografie lo documentano, sono un'emanazione anche dei sentimenti raggelati, dal Dopoguerra a oggi, degli abitanti.

Come dicevamo le fotografie sono analogiche, in formato $6 \times 6$, scattate con una macchina fotografica fabbricata negli stessi anni in cui si edificavano i luoghi ritratti. Questo può sembrare un vezzo, ma del resto, anche alcuni cuochi non rinunciano a cucinare in determinate pentole che assicurano una cottura migliore rispetto a pentole contemporanee.

La carta usata per il testo è uguale alla carta usata per le fotografie. Volevamo che, anche a livello percettivo non ci fosse un salto, una differenza tattile tra testo e immagine. Quindi, un fototesto, come Condominio Oltremare, 
si basa su un triplice ritmo: del testo, delle fotografie, e del sottile rapporto tra testo e fotografie.

Flashover, edito nel settembre 2020 da Einaudi, ha un personaggio protagonista del testo - l'incendiario della Fenice di Venezia -, definito da Falco «il cugino padrone», in minuscolo. È quindi un personaggio con un non-nome, quasi al di sotto della soglia di personaggio, alla ricerca, vana, di un'identità. $\mathrm{Al}$ «cugino padrone» si alterna un secondo personaggio, interpretato da Falco, che abita la parte visiva, attraversa mascherato il testo e rappresenta l'espressione concreta degli stati d'animo di protagonisti, autori e spettatori. L'unione tra testo e immagine è il luogo in cui i due personaggi si incontrano. La maschera è il tramite tra il personaggio del lavoro di Ragucci e «il cugino padrone»; per giungere alla verità dell'opera, sono state necessarie due maschere: una semantica e una visiva.

Mentre ogni singola fotografia pulsa, a volte in modo allarmante, da sola, il significato dell'insieme coesiste solo quando tutte le sue parti e le connessioni subliminali sono state completamente assorbite, anche grazie alla sequenza, all'insistenza, alla serialità. Il personaggio in maschera, così come «il cugino padrone», è alla ricerca di un'identità. Il lavoro visivo presente in Flashover, intitolato Alfabeto della distruzione, è senz'altro anche un rimando alla tradizione novecentesca, ai lavori in maschera di Meatyard e Wojnarowicz; o al più recente film-documento di Huyghe, in cui una scimmia, con una maschera umana, si aggira in un edificio disabitato, in una dimensione post-apocalittica. Tuttavia in Flashover non c'è alcuna dimensione post-apocalittica, non serve un evento traumatico, siamo già entrati in una dimensione post-umana di cui l'uomo in maschera è l'emblema. A differenza delle maschere grottesche e mostruose di Meatyard, o della maschera-Rimbaud usata da Wojnarowicz a New York, la maschera del personaggio di Flashover è poco caratterizzante, è banale, la maschera globale, la maschera del commercio, la maschera del denaro, la maschera dei flussi di denaro che impoveriscono il mondo. La maschera è la matrioska del volto, di un, scrive Falco, «invincibile teschio» (Falco - Ragucci 2020: I67). È nella impercettibile distanza tra volto e maschera che si annida l'essenza del nostro vivere. È lì che si annida il desiderio, la mancanza, la quantità di denaro più o meno grande, ma è lì che accade qualcosa. Così come è accaduto nel desiderio continuo del «cugino padrone». Così come accade nell'immagine.

Utilizzare il linguaggio della fotografia predispone a confrontarsi con parte della sua storia ed è oramai inevitabile; le voci degli altri emergono in continuazione, sebbene in una forma che non vuole più appartenere alle gallerie d'arte. Del resto, se l'arte esiste, seguendo l'esempio di Godard, ci ricorda che la forma dice sempre qualcosa dell'epoca che si sta vivendo, ed è la forma la sola cosa che sopravviverà. Merleau-Ponty nel descrivere l'opera d'arte come una manifestazione sensibile in cui s'intrecciano immanenza e 
trascendenza, conferma che il problema della rappresentazione per immagini si esaurisce a vantaggio della risonanza dell'immagine sull'Io, e questa verità paradossale dell'arte si rivela solo a colui che, con la sua percezione si lascia pervadere da un'esperienza in cui lo sguardo e la cosa vista perdono i loro reciproci contrassegni esteriori: qualità, luce, colore, profondità sono davanti a noi soltanto perché risveglino un'eco nel nostro corpo, perché esso li accolga. Questa volta i superstiti siamo noi, e mai come adesso, sappiamo di essere intrappolati in una vorticosa eternità che finisce.

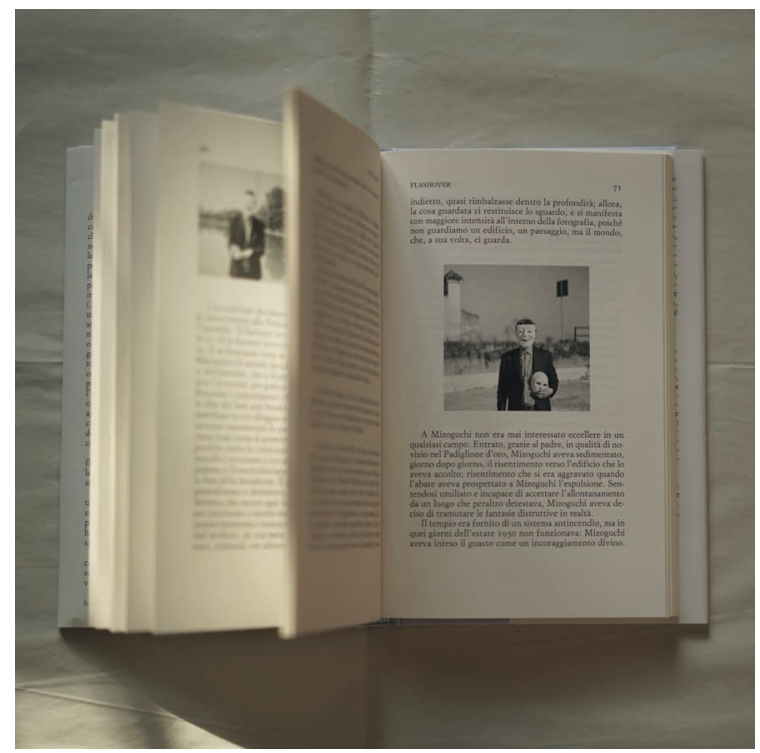

\section{Bibliografia}

Abbott, Berenice, A Guide to Better Photography, New York, Crown Publishers, I94I.

Agee, James - Evans, Walker, Let Us Now Praise Famous Men [194I], trad. it. Sia lode ora a uomini di fama, Milano, Il Saggiatore, 2002.

Dick, Philip K., Ubik, postfazione e cura di C. Pagetti, trad. di P. Prezzavento, Roma, Fanucci, 2007.

Dine, Jim, The Photographs, So Far, Göttingen, Steidl, 2003.

Evans, Walker, Signs, with an essay by A. Codrescu, London, Thames and Hudson, 1998.

Falco, Giorgio, L’ubicazione del bene, Torino, Einaudi, 2009.

Falco, Giorgio - Ragucci, Sabrina, Condominio Oltremare, Roma, L'orma, 2014. -. Flashover. Incendio a Venezia, Torino, Einaudi, 2020. 
Handke, Peter, Saggio sul luogo tranquillo, trad. di A. Iadicicco, Parma, Guanda, 2014 .

Jung, Carl Gustav, Il libro rosso. Liber novus, a cura di S. Shamdasani, Torino, Bollati Boringhieri, 2012.

Kallir, Alfred, Segno e disegno. Psicogenesi dell'alfabeto, Milano, Spirali/Vel, I994.

McCausland, Elizabeth, «Photographic Books», The Complete Photographer, 43, January 1942, pp. 27-85.

Mikhailov, Boris, Unfinished Dissertation (or Discussions with Oneself), Göttingen, Scalo, 1998.

Ragucci, Sabrina, Il medesimo mondo, Torino, Bollati Boringhieri, 2020.

Sander, August, Wesen und Werden der Photographie, V, Die Photographie als Weltsprache, manoscritti inediti di sei conferenze tenute a Westdeutscher Rundfunk (WDR), I93I (August Sander Archiv, Photographische Sammlung/SK Stiftung Kultur, Köln); ora «Die Photographie als Weltsprache», in Id., Sehen, Beobachten, Denken. Photograhien, mit Texten von A. Sander und G. Conrath-Scholl, München, Fondation Henri Cartier-Bresson Schirmer/Mosel Verlag, 2009, pp. 25-32.

Strand, Mark, «Note sul mestiere della poesia», in Tutte le poesie, trad. di D. Abeni con M. Egan, Milano, Mondadori, 2019, pp. XVII-XIX.

Whitman, Walt, Foglie d'erba, trad. di E. Giachino, Milano, Mondadori, 1977. 